\title{
What is the potential of patient shadowing as a patient-centred method?
}

\section{Introduction}

A recently published study by Yanes et al. ${ }^{1}$ suggests that observation in clinical environments may have an especially valuable role in capturing the organisational and situational factors that shape clinical processes. In data dense and high-risk environments, observation sheds light on the specific sub-processes of complex clinical activities (e.g. information transfer, communication patterns, distractions) and their effects on patient care. Observation also captures aspects of patients and providers' experiences that may be missed by traditional research (e.g. interviews or questionnaires) and that people may find difficult to articulate. Yanes et al. discuss the different potentialities of in-person and video recorded observations, but the range of observational techniques that can be adopted merit further consideration. In this article I propose that observational methods in healthcare could, building on recent work, ${ }^{2-4}$ be extended to include patient shadowing. Such an approach may have particular value as a patient-centred method, ${ }^{3-6}$ yet has remained remarkably neglected.

Patient-centred care has been defined as an approach that seeks to explore patients' desires, preferences, values, and concerns, with the aim of empowering them to make decisions that best fit their individual needs. ${ }^{7}$ Identified by the Institute of Medicine as one of the six fundamental elements of high-quality care, ${ }^{8}$ it is now considered an essential component of care delivery. ${ }^{7,8}$ But despite broad agreement on the principle, precisely what patient-centred should look like in practice has thus far escaped consensus. ${ }^{2,9,10}$ It has multiple and sometimes conflicting meanings for different care providers, ${ }^{9}$ and patients themselves may value aspects of their health experiences that receive little attention in research and practice. Official policies on patient-centredness often co-exist with practices that relegate patients into a passive and dependent position. ${ }^{2}$ Since important questions remain unanswered regarding how to conceptualise and accomplish patient-centred care, healthcare systems may lack concrete direction for action. This problem may be compounded by the limited methods used to gain insights into patient-centeredness. Patient shadowing may have an especially valuable role in addressing this deficit.

'Shadowing' means following individuals throughout their daily actions, observing and conversing with them when possible without disrupting their normal flow of activities. Ethnographers recommend using this technique in contexts where traditional static observation is not well-suited to capturing the timespace dynamics of the activities addressed. ${ }^{11}$ Shadowing patients in particular allows the observer to witness clinical work and care processes from their point of view. As a research method, it may contribute to patient-centredness in both conceptual and practical ways.

Shadowing allows collection of in-depth qualitative data concerning patients' and families' subjective care experiences, as well as broader qualitative and quantitative evidence of how care pathways unfold in time and space within a network of care providers. Thus, the method may enable a more nuanced and organisationally-aware conceptualisation of patient patient-centredness, beyond the well-studied patientdoctor relationship, to include the structural and cultural factors that shape patient care experiences (e.g. waiting times, ease of access to information, breakdowns in communication among practitioners, etc.). 
But patient shadowing can also contribute to more practical improvement goals. It may aid the diagnosis of aspects of routine care practice that hinder patient-centredness and the identification of actionable opportunities to improve care delivery. ${ }^{3,4,12}$ Examples include the strengthening of effective transitions between different healthcare providers/services ${ }^{12}$, the creation of a more efficient prioritisation system for treating patients within specific care pathways ${ }^{13}$ and the delivery of better education to patients in the discharge phase in order to reduce avoidable return visits. ${ }^{6,13}$ Other improvement actions may be simpler and less expensive, but nevertheless capable of improving patients' care experience. For example, having learned that patients going to theatre were often cold from fasting, a NHS service decided to invest in a blanket warmer. ${ }^{5}$ The benefits of patient shadowing for service improvement have been recently highlighted, among others, by the Patient and Family Centered Care Innovation Center.

\section{Challenges}

Gaining such a privileged perspective on patients' experience is not without costs, including the challenge of simultaneously observing and recording multiple types of data (conversational, observational) while being cognizant of one's influence on the field. Though anecdotal evidence suggests that interest towards patient shadowing is increasing rapidly and some guides to good practice do exist, ${ }^{14}$ thorough examination of its practical, methodological, and ethical challenges is still lacking. Issues remain to be settled in relation to the status of shadowing as a relational method, what shadowers do, the role of the patient in the planning and conducting of shadowing, and how to convert the findings into impacts on patients' care experiences. Selected insights from ethnography $y^{11,15,16}$ and current debates on co-production ${ }^{17,18}$ could shed further light on how these challenges might optimally be addressed.

A relational method. Ethnographers suggest that the "site" of shadowing research is the interaction between researcher and participant; ${ }^{15}$ shadowing thus requires significant self-reflexivity and awareness of one's positionality on the researcher's part. Loosely, this means recognising how one's views, beliefs, social role, and power affect relationships with participants as well as research outcomes. Although seemingly abstract, these concepts may have very pragmatic consequences for patient shadowing. It may be especially difficult for less experienced shadowers to regulate their position with respect to patients and families. Excessive proximity may cause discomfort: patients may perceive shadowers as intrusive and not respectful of their privacy, or may, alternatively, consider them as confidants or advocates. Observing patients from a distance is not a solution either: people may feel 'spied on' when most vulnerable, and shadowers may fail to fully understand patients' care experiences from their point of view. ${ }^{16}$ Balancing proximity and distance is only one example of the challenges that shadowers may face in the field. Shadowing is a relational exercise: it relies on the willingness of participants to take part in it and on a trusting relationship between researchers and participants, which requires time, experience, and frequently renewed agreements. Providing shadowers with selected insights from ethnography may prove beneficial to their self-awareness and ability to conduct shadowing in an informed and reflexive way.

What do shadowers do? One of the most discussed aspects of observational methods is the degree to which observers do and should intervene in real-time. In more traditional observations, both the researcher and the participants may be able to experience time alone and to "hide" from each other. But shadowing involves a more intimate experience - what Czarniawska defines a "peculiar twosome". ${ }^{11}$ Most ethical guidelines suggest that observers should not participate in the clinical process, but in extreme circumstances they may be ethically bound to intervene. ${ }^{1}$ The possibility of intervening becomes acute when shadowers know more about what is going on than do patients and families. They may find themselves wondering whether it is their role to provide more information, or to encourage 
patients/families to speak up and require more information. In some circumstances, by strictly adhering to a non-reactive position, shadowers may be perceived as exploiting patients for research purposes. These issues raise questions as to whether shadowers should act narrowly as observers or become "buffers" or brokers between patients, families, and clinicians, and consequently about how to maintain the appropriate boundary between research and advocacy.

When and what to shadow. One little-discussed aspect of patient shadowing relates to when to shadow patients and what to observe: selecting the timeframe for shadowing and drawing the boundaries of the patient's care journey may not be straightforward. Shadowing patients during their entire trajectory (from admission to discharge and going home from hospital, for example) may be informative but also costly and unsustainable, and thus shadowing only selected care experiences (e.g. a visit to an outpatient appointment) may seem a practical alternative. However, letting researchers decide ex ante which part of the care process is more worthy of observation may neglect aspects of care which patients consider important. Thus, involving patients in establishing the aims and boundaries of shadowing is essential, but it requires awareness of the broader challenges of co-production in healthcare research and services, including those of hierarchy and diversity. ${ }^{17}$ The criteria used to involve patients in every step of shadowing should be carefully considered and reported in full.

Uncomfortable feedback. Patient shadowing may reveal gaps between aspirations of patient-centredness and what really happens. Care delivery processes may run with more consideration for the interests of healthcare staff than the patients' needs, and patients may be left with little access to information and opportunities to participate in planning their care. In public healthcare services tensions may emerge between the need to deliver better care to individual patients and the shrinking resources available. Ultimately, for patient shadowing to produce relevant knowledge, shadowers should be able and willing to provide feedback in actionable ways and to manage relationships effectively, especially when offering uncomfortable news. ${ }^{18}$ Yet this may be especially controversial for shadowers who are employed by the same organisation where they conduct shadowing. Healthcare services, for their part, must be committed to and capable of implementing the changes needed to achieve patient-centredness. Current debates suggest that such co-production is often hindered by professional-centric cultures and easily loses priority in the face of productivity pressures. Putting the knowledge generated by patient shadowing into practice thus remains a major challenge. 
1. Be reflexive of your role as a shadower: how is your social and professional background perceived by patients and families? How does it affect their freedom to speak with you and with others?

2. Exercise your ability to detect 'weak signals' when regulating proximity and distance: patients and families may express their desires in indirect ways.

3. Make it clear that your presence as a shadower will not influence the care the patient receives. ${ }^{3}$

4. Look for patients' input when establishing the time-frame for shadowing.

5. Collect data extensively. Observe how the patient is treated by care providers, what information he/she receives, what organisational/contextual elements affect the care process, what the patient appreciates and what he/she perceives as problematic. ${ }^{3,14}$

6. Establish criteria for involving patients: to what extent will they participate in designing and conducting shadowing, analysing the data, and suggesting improvement opportunities?3-5

7. Patients can withdraw from the research or renegotiate conditions for participation at any time.

8. Engage clinicians. ${ }^{4}$ Explain the aims of patient shadowing to all the care providers involved in the care pathway, negotiate access with them, and invite them to indicate areas for improvements.

9. Patients should be invited to indicate whether the shadowing has captured all the important aspects of their care experience. If not, the shadowing exercise may be extended/modified.

10. Negotiate the modalities for feeding back the findings to patients as well as care providers.

\section{Conclusions}

Yanes et al. have emphasised the value of observations in healthcare improvement. The potential of a specific observational method - i.e. patient shadowing - is now being recognised as an important way of advance patient-centred care both conceptually and practically. Yet it is clear that patient shadowing is not a 'quick and simple' approach. Fundamental questions remain to be addressed, such as those relating to the ability of patients to determine the care experience to be shadowed, to choose the observer, and to contribute to data analysis in order to suggest possible improvements. Examination of these aspects is key to avoid conflating patient-centredness with aspects of the care process that patients perceive as secondary. The role of the shadowers is also complex, involving dilemmas about how to achieve an indepth understanding of patients' experience while respecting their privacy. These challenges are, to some extent, inherent to the practice of shadowing patients, regardless of the specific aims of the research (conceptual or practical). Some principles of good practice can be identified (Box 1).

As is often the case with new research methods, the key to good practice is self-reflexive practice. Fuller, more nuanced, and more reflexive research accounts of the experience of shadowing from multiple perspectives - the observers, patients, and caregivers - are much needed. Strengthening the collaboration between clinicians and social scientists may lead to designing more comprehensive training programs, attentive to the methodological challenges and potential ethical dilemmas inherent to this method, though not limiting its use solely to ethnographers. 


\section{References}

1. Yanes AF, McElroy LM, Abecassis ZA et al. Observation for assessment of clinician performance: A narrative review. BMJ Qual Saf. 2016;25(1):46-55.

2. Liberati EG, Gorli M, Moja L et al. Exploring the practice of patient centered care: The role of ethnography and reflexivity. Soc Sci Med. 2015;133:45-52.

3. NHS Institute for Innovation and Improvement. Quality and service improvement tools, patient perspectives. http://www.institute.nhs.uk/quality and service improvement tools/quality and service improvement tools/patie nt perspectives.html. Updated 2008. Accessed 04/07, 2016.

4. DiGioia AM, Lorenz H, Greenhouse PK et al. A patient-centered model to improve metrics without cost increase: Viewing all care through the eyes of patients and families. J Nurs Adm. 2010;40(12):540-546.

5. Shaw J, Pemberton S, Pratt C et al. Shadowing: A central component of patient and family-centred carefamilies. Nurs Manage. 2014;21(3):20-23.

6. Hawkes N. Patients' actual care pathways often differ markedly from doctors' perceptions. BMJ. 2013;347.

7. Stewart M, Belle Brown J, Weston WW et al. Patient-centered medicine: Transforming the clinical method. London: Sage Publications; 1995.

8. Institute of Medicine. Crossing the quality chasm: A new health system for the 21st century. In: Committee on quality of healthcare in america. Washington: National Academies Press; 2001.

9. Gillespie R, Florin D, Gillam S. How is patient-centred care understood by the clinical, managerial and lay stakeholders responsible for promoting this agenda? Health Expect. 2004;7(2):142-148.

10. Kitson A, Marshall A, Bassett $\mathrm{K}$ et al. What are the core elements of patient-centred care? A narrative review and synthesis of the literature from health policy, medicine and nursing. J Adv Nurs. 2013;69(1):4-15.

11. Czarniawska-Joerges B. Shadowing: And other techniques for doing fieldwork in modern societies. Copenhagen Business School Press DK; 2007.

12. DiGioia AM, Greenhouse PK, Chermak T et al. A case for integrating the patient and family centered care methodology and practice in lean healthcare organizations. Healthcare. 2015;3(4):225-230.

13. National Association for Healthcare Quality. Shadowing improves patient care experience and communication between families and clinicians. National association for healthcare quality Web site.

http://www.nahq.org/uploads/Greenhouse talk news release.pdf. Updated 2015. Accessed 04/07, 2016.

14. DiGioia AM, Shapiro E. Shadowing guide. View all care through the eyes of patients and families. The Patient and Family Centered Care Innovation Center of UPMC Web site. http://www.pfcc.org/newpfcc/wpcontent/uploads/2015/02/ShadowGuideWeb new.pdf. Updated 2013. Accessed 04/07, 2016.

15. Gill R. The shadow in organizational ethnography: Moving beyond shadowing to spect-acting. Qualitative Research in Organizations and Management: An International Journal. 2011;6(2):115-133.

16. Dixon-Woods M. What can ethnography do for quality and safety in health care? Qual Saf Health Care. 2003;12(5):326-327.

17. Batalden M, Batalden P, Margolis P et al. Coproduction of healthcare service. BMJ Quality \& Safety. 2015. 
18. Brewster L, Aveling E, Martin G et al. What to expect when you're evaluating healthcare improvement: A concordat approach to managing collaboration and uncomfortable realities. BMJ Quality \& Safety. 2015. 